“C 2019 IEEE. Personal use of this material is permitted. Permission from IEEE must be obtained for all other uses, in any current or future media, including reprinting/republishing this material for advertising or promotional purposes, creating new collective works, for resale or redistribution to servers or lists, or reuse of any copyrighted component of this work in other works." 


\title{
Spatially Filtered Low-Density EMG and Time-Domain Descriptors Improves Hand Movement Recognition
}

\author{
Ahmed A. Al Taee, Rami N. Khushaba, Senior Member, IEEE, Adel Al-Jumaily, Senior Member, \\ IEEE
}

\begin{abstract}
Surface Electromyogram (EMG) pattern recognition has long been utilized for controlling multifunctional myoelectric prostheses. In such an application, a number of EMG channels are usually utilized to acquire more information about the underlying activity of the remaining muscles in the amputee stump. However, despite the multichannel nature of this application, the extracted features are usually acquired from each channel individually, without consideration for the interaction between the different muscles recruited to achieve a specific movement. In this paper, we proposed an approach of spatial filtering, denoted as Range Spatial Filtering (RSF), to increase the number of EMG channels available for feature extraction, by considering the range of all possible logical combinations of each $\boldsymbol{n}$ channels. The proposed RSF method is then combined with conventional time-domain (TD) feature extraction, as an extension of the conventional single channel TD features that are heavily considered in this field. We then show how the addition of a new feature, specifically the minimum absolute value of the range of each two windowed EMG signals, can significantly reduce the different patterns misclassification rate achieved by conventional TD features (with and without our RSF method). The performance of the proposed method is verified on EMG data collected from nine transradial amputees (seven traumatic and two congenital), with six grip and finger movements, for three different levels of forces (low, medium, and high). The classification results showed significant reduction (nearly $10 \%$ for some individual TD features and $5 \%$ for combined TD features) in classification error rates compared to other methods.
\end{abstract}

\section{INTRODUCTION}

The Electromyogram (EMG) signals recorded from the muscles in the residual limb of an individual with amputation has been shown as an effective source of neural control information for powered prostheses [1]. Despite the success achieved in offline EMG pattern recognition studies, the clinical translation of offline findings has been greatly limited. Part of this problem is related to the fact that offline findings with intact-limbed subjects may not translate to clinical applications for subjects with different levels of amputations, since the potential EMG recording sites are limited while the number of functions to control is increased. One possible solution is to increase the number of EMG channels by targeted muscle reinnervation (TMR) surgery in combination with high-density EMG (HD-EMG), i.e., multichannel EMG recorded using $2 \mathrm{D}$ grids of closely spaced sEMG electrodes [2]. However, the high-information density in the reinnervated

Ahmed A. Al Taee, Rami Khushaba, and Adel Al-Jumaily are within the Faculty of Engineering and Information Technology (FEIT), University of Technology, Sydney (UTS), 15 Broadway, Ultimo 2007, NSW, Australia. (email: Ahmed.A.Ahmed@student.uts.edu.au, Rami.Khushaba@uts.edu.au , Adel.aljumaily@uts.edu.au ). muscles was reported to be a potential source of misclassification errors. The use of spatial filtering (SF) [3] was then justified in studies with both HD-EMG with TMR and HD-EMG without TMR to capture the focal muscle EMG activity, improve the spatial resolution of EMG signals, and improve the overall classification accuracy of identifying intended movements $[4,5]$. A second solution to increase the number of EMG channels also utilizes SF, but to combine the EMG signals of several recording channels to form new logical combinations of EMG signals from which to extract the EMG features of interest. Khushaba et al [6] recently presented one such approach while suggesting some novel time-domain features and testing on studies with high- and low-density EMG [6]. It was shown that a significant reduction in classification error rates can be achieved when using SF with carefully selected temporal descriptors. It was also shown, in the context of their experiments on HD-EMG datasets, that adding more channels allowed small windows size to be utilized with a combination of $>32$ channels to achieve less than $10 \%$ error on average. On the other hand, Menon et al [7] further investigated the effect of temporal and spatial information provided to the classifier while varying the analysis window length, window overlap, and the number of HD-EMG channels (with some conventional time-domain descriptors). It was shown that there is a complex interplay between these factors, and that the type of limb deficiency dictates the benefits of adding more electrode channels. Hence, while the importance of applying SF in studies with HD-EMG was shown in many studies, the study of the impact of SF on the feature extraction step with low-density EMG still requires further investigations. The main justification is that most practical prostheses controllers can practically only accommodate a small number of EMG channels and therefore studying the methods of further enhancing the EMG pattern recognition under these conditions is of significant importance to achieve clinically viable applications of this technology.

To continue upon the literature and our previous findings in this field of research [6], we investigate in this paper the impact of adding more channels by SF with conventional and new time-domain features in a problem with low-density EMG under varying force levels (low, med, and high). This allows more feasible clinical implementations as it employs a small number of physical channels while utilizing SF to create logical combination of these channels to increase the amount of neural information that we can extract. The impact of this approach is also shown in comparison to utilizing the same 
time-domain features without SF under varying force levels. Our approach could also be a step towards the natural control in everyday applications where a subject could use low or medium force levels to achieve the intended tasks.

\section{Methodology}

\section{A. Spatially-Filtered Time-Domain Feature Extraction}

A number of spatial filters were proposed for EMG signal smoothing in the literature, including for example single and double differential (SDF and DDF) spatial filters for special electrode arrangements [8]. However, our approach is not aimed at smoothing the original signals but to create a new set of logical signals based on combination of the original and then extracting features from the generated logical signals rather than the original EMG signals. For this specific purpose, we investigated a number of options including SDF and DDF, however, within the experiment and data at hand, we found that a proposed range spatial filter provided significantly lower classification error rates. The proposed range spatial filter (RSF) accepts as an input all possible combinations of two (or more) channels out of the total $N C$ channels in the problem at hand and outputs the range of the two (or more) signals across each sample. For example, if the $k$ samples of the first channel (denoted as $c h_{A}$ ) and the second channel (denoted as $c h_{B}$ ) are fed to this filter then the output of RSF will be another set of $k$ samples as shown in Fig.1 and given below

$$
c h_{C}=\left[\max \left(c h_{A}, c h_{B}\right)-\min \left(c h_{A}, c h_{B}\right)\right]
$$

As a result of the above, a new set of a total of ( $(N C \times \mathrm{x} N C)$ $N C) / 2$ logical channels are created from which we extract the time-domain (TD) features. In terms of feature extraction, TD features have been heavily utilized in EMG pattern recognition systems like those proposed by Hudgins et al [9] and many others [10], as TD features can be used in real-time application, are easy to implement, and have low computational complexity associated with a good pattern recognition performance [6]. Among the most utilized TD features in the literature are [9,10]: Mean Absolute Value (MAV), Root Mean Square (RMS), MMAV Slope (MAVS), Waveform Length (WL), Zero Crossings (ZC), Integral Absolute Value (IAV), and Hjorth TD features (HTD) [6]. Phinyomark et al also looked at more than 50 features for this purpose [10]. The proposed RSF method can be applied with any of these feature extraction methods. Additionally, a new feature which is the minimum absolute value (MIN) of the range generated signals has been utilized in our experiments with different combination of TD features from the literature. In the experiments we show how the MIN feature can add a significant value in reducing the classification error rates across all force levels.

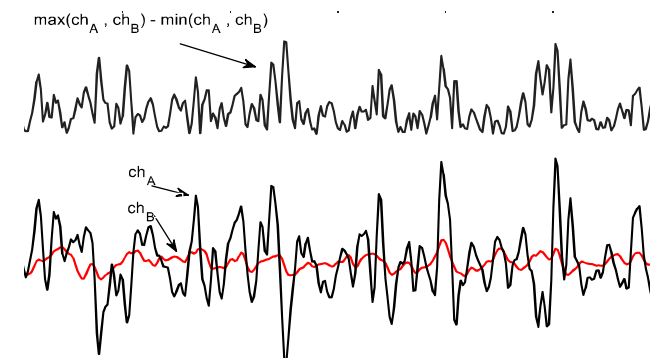

Figure 1. RSF example depicting the generation of new signal from two channels EMG signals.

\section{B. Data Collection}

The EMG dataset included data collected from 9 transradial amputees (seven traumatic and two congenital) with unilateral amputation as per earlier description provided in [11]. A custom-build acquisition system was used to acquire the EMG data from 8 channels at a sampling rate of $2000 \mathrm{~Hz}$. A virtual Instrument (VI) implemented in LABVIEW (National Instruments, USA) was used for signal acquisition and display. This was used by the amputees to help produce the needed force level. The intact-hand was used by the amputees to help them imagine the needed movement with the required force level. Six movements including different grip and finger movements were investigated, these movements were: 1) Thumb flexion; 2) Index flexion; 3) Fine pinch; 4) Tripod grip; 5) Hook grip (hook or snap); 6) Spherical grip (power). After electrodes placement, each amputee was asked to examine the EMG signals on the screen in real-time and familiarize themselves with the changes in force of contraction for different movement. For each gesture, the amputee produced three force levels: low, med, and high with the help of visual feedback from the intact limb as shown in Fig.2. For each force level, 5 to 8 trials were recorded with each trial lasting for almost 8 to $12 \mathrm{sec}$. Thus, the total number of trials performed by each amputee was equal to the number of movements $\times$ number of force levels $\times$ number of trials for each movement. The EMG datasets for the first 6 amputees TR1-TR6 (Transradial 1 to 6) were collected at the Artificial Limbs and Rehabilitation Centers in Baghdad and Babylon, Iraq, while the EMG datasets for TR7 (Transradial7), CG1 (Congenital 1) and CG2 (Congenital 2) were collected at Plymouth University, UK. The local ethical committee at the School of Computing and Mathematics, Plymouth University approved this research. Eight pairs of $\mathrm{Ag} / \mathrm{AgCl}$ electrodes (Tyco healthcare, Germany) connected to a differential amplifier were placed around the left stump in one or two rows for all amputees apart from CG2 where the electrodes were placed on the right stump.

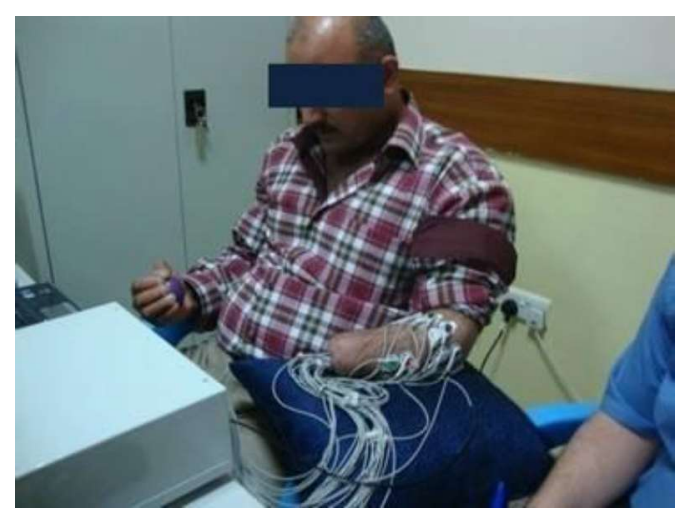

Figure 2. Amputee TR5 executing the protocol for recording different forcelevels. He used the VF and intact limb to produce the spherical grip, as it can be seen in the picture.

\section{EMG Pattern Recognition}

In the experiments, an overlapping windowing scheme was utilized to extract the proposed features, with an analysis window size of $150 \mathrm{~ms}$ and an increment of $50 \mathrm{~ms}$. MATLAB ${ }^{\circledR} 2018$ b software (Mathworks, USA) was used to 
perform the analyses. In all analysis, features extracted from the first 3 trials of each hand movement, for each force level, were assigned to the training set, while features extracted from the remaining trials were assigned to the testing set. A number of experiments were carried out to evaluate the performance of the RSF method with different sets of TD features, with and without the MIN features. In this regards, and considering the EMG signals collected under different force levels, we performed the following analysis.

- Firstly, we evaluated the performance of the proposed RSF method with a basic set of individual TD features and show that RSF can add significant reductions in classification error rates across almost all features.

- Secondly, we show that by using RSF with MIN features in combination with many reported TD feature sets from many research groups that the combination of TD features with RSF+MIN can effectively reduce classification errors across all force levels. Among the methods considered here were the combination of $\mathrm{MAV}+\mathrm{ZC}+\mathrm{SSC}+\mathrm{WL}$ [9], $\mathrm{MAV}+\mathrm{ZC}+\mathrm{SSC}+\mathrm{WL}[12], \mathrm{SSC}+\mathrm{ZC}+\mathrm{WL}$ combined with AR+RMS [13], and $\mathrm{SSC}+\mathrm{WL}+\mathrm{ZCC}+\mathrm{SS}$ combined with HTD+AR [14].

The dimensionality of the extracted features from all of the considered features combinations was reduced using Spectral Regression (SR) dimensionality reduction method proposed by Cai et al. [15]. SR maps the original feature set into a new domain with $c-1$ features only, with $c$ being the number of classes. Finally, in terms of classification accuracy, we have utilized the well-known Linear Discriminant Analysis (LDA) as it has proved its effectiveness in EMG pattern recognition in many studies and is well utilized in this field of research with results comparable to Support Vector machine in many studies [1].

\section{RESULTS AND DISCUSSION}

The average classification error rates of many individual TD features were evaluated across all EMG channels, and averaged across all force levels, with and without the application of our RSF method as shown in Fig. 3. It should be noted here that we had 8 channels of EMG data for the no RSF results, while with the RSF method we ended up with 28 logical combinations of these channels (denoted as RSF results). Hence, SR dimensionality reduction was applied on all features (no RSF and RSF) for consistency before submitting the features to the LDA classifier. For all of the results in Fig. 3, ANOVA test with Bonferroni correction was applied at a significance level of 0.05 with all $p$-values being $<<0.01$, except for Hjorth parameters that had $p<0.02$, which clearly indicates the benefit of applying the RSF method at the individual features level.

In the second part of the experiments we tested the classification error rates achieved by several combinations of TD features as reported in the literature as shown in Fig. 4.

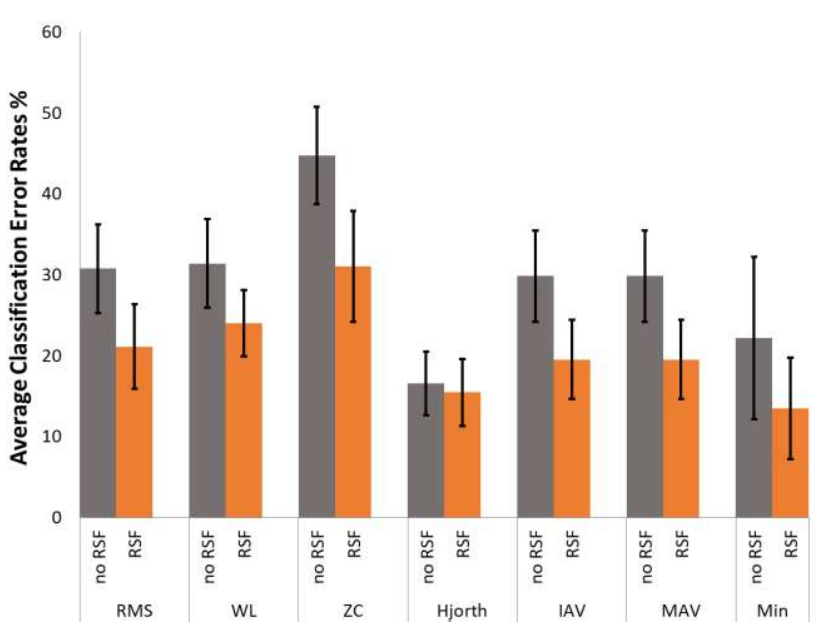

Figure 3. Individual TD classification erro rates with and without the proposed RSF method averaged across all force levels.

We show that on average across all amputees, these classification error rates dropped significantly across each of the force levels when using the aforementioned TD feature sets with the RSF method, with the addition of our proposed MIN feature further reducing the classification errors. It can also be clearly seen from the results in Fig. 4 that it is desirable to have the data collected at low and medium force levels as the classification error rates for the high force level were higher than that achieved with low and med force levels. This is also intuitive as generally speaking, the high force is difficult to perform for an amputee person since it requires a lot of effort from them as they need to employ visual feedback from the intact limb during the high force level movements. Additionally, producing a high force level and maintaining it for long time might produce fatigue since the amputee persons have not used their stump muscles for long time. This might explain why the error rates were much higher for the high force levels than the low and medium levels of force.

\section{CONCLUSION}

In general, these results suggest that range spatial filtering of EMG recordings may further improve the EMG-based interface for robust, multifunctional control of artificial arms. It is also important to mention here that many TD features (individually and combined in sets) presented earlier in the literature are only focusing on the temporal part of the feature extraction as such methods do not look at the relation between the EMG signals from the different channels which the RSF method can capture (to the extent suggested in this paper). In comparison to conventional TD feature extraction, our combined RSF+TD method also looks at how the EMG characteristics of the different channels change across the different movement and force levels and was therefore more capable of further reducing the classification error rates. This in turn proves our main point of the benefits of extending the feature extraction process by employing spatial filtering, not only in HD-EMG studies [4], but also in low-density EMG studies to extend the amount of available information and extract the most discriminant features from the time-domain directly.

It is also important to mention here that while the current analysis in this paper considered RSF filters with only two 
input channels (mainly for simplicity and initial proof of concept reasons), however, our approach can be applied across any number of input channels. Our research continues to explore the benefits associated with the spatial filtering approach across larger problems (HD-EMG and LD-EMG) with more inputs and across more dynamic factors i.e., not only varying force levels but also varying limb positions, rotations and many other factors.

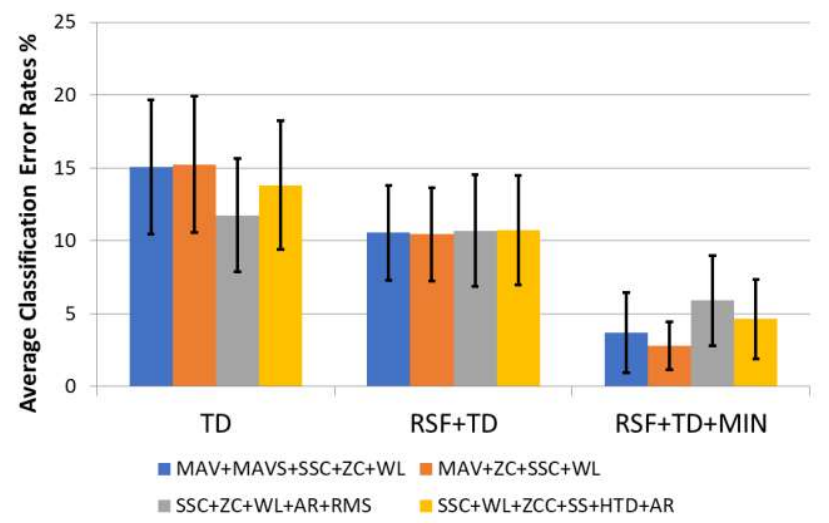

(a). Low Force level

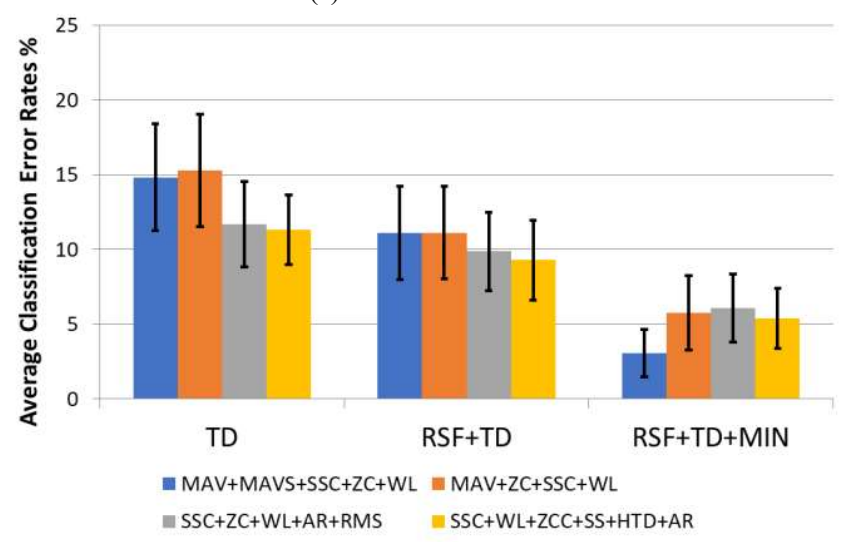

(b). Med Force level

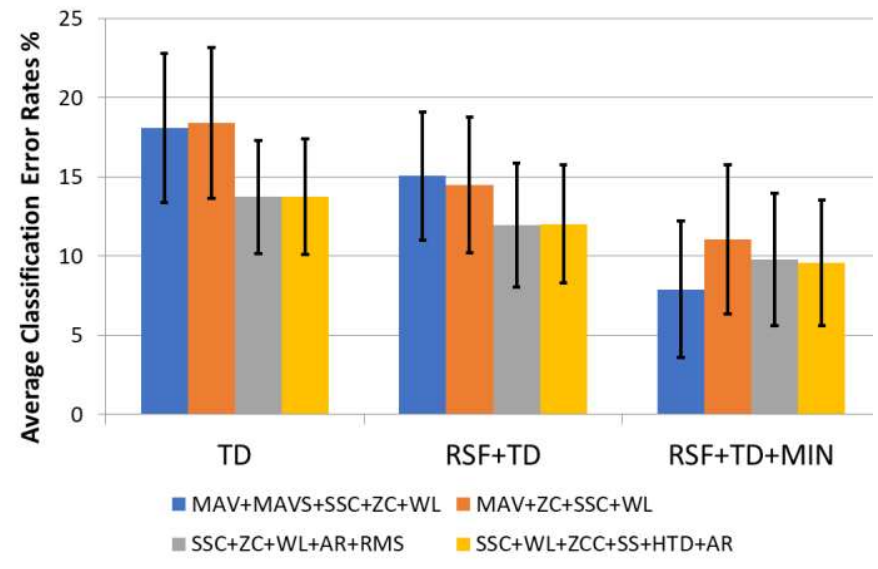

(c). High Force level

Figure 4. Different TD feature sets' classification erro rates with and without the proposed RSF method and the MIN feature.

\section{ACKNOWLEDGMENT}

The authors would like to acknowledge the use of the
EMG datasets from a previous study by the second author and Dr. Ali Al-Timemy from Plymouth University and the University of Baghdad.

\section{REFERENCES}

[1] M. A. Oskoei and H. Hu, "Myoelectric Control Systems-A Survey," Biomedical Signal Processing and Control, vol. 2, no. 4, pp. 275-294, 2007.

[2] H. Huang, P. Zhou, G. Li, T.A. Kuiken, "An analysis of EMG electrode configuration for targeted muscle reinnervation based neural machine interface". IEEE Transactions on Neural Systems and Rehabilitation Engineering, vol 16, pp. 37-45, 2008.

[3] C. Disselhorst-Klug, J. Silny, and G. Rau, "Improvement of spatial resolution in surface-EMG: a theoretical and experimental comparison of different spatial filters", IEEE Transactions on Biomedical Engineering, vol. 44, pp. 567-574, 1997.

[4] H. Huang, P. Zhou, G. Li, and T. Kuiken. "Spatial filtering improves EMG classification accuracy following targeted muscle reinnervation", Annals of Biomedical Engineering, vol. 37, no. 9, pp. 1849-57, 2009.

[5] M. Jordanić, M. Rojas-Martínez, M. A. Mañanas, J. F. Alonso, and H. R. Marateb, "A Novel Spatial Feature for the Identification of Motor Tasks Using High-Density Electromyography", Sensors (Basel), vol 17, no. 7, pp. 1597, 2017.

[6] R. N. Khushaba, A. H. Al-Timemy, A. Al-Ani and A. Al-Jumaily, "A Framework of Temporal-Spatial Descriptors-Based Feature Extraction for Improved Myoelectric Pattern Recognition," in IEEE Transactions on Neural Systems and Rehabilitation Engineering, vol. 25, no. 10, pp. 1821-1831, 2017.

[7] R. Menon, G. Di Caterina, H. Lakany, L. Petropoulakis, B. A. Conway and J. J. Soraghan, "Study on Interaction Between Temporal and Spatial Information in Classification of EMG Signals for Myoelectric Prostheses," in IEEE Transactions on Neural Systems and Rehabilitation Engineering, vol. 25, no. 10, pp. 1832-1842, Oct. 2017.

[8] M. Ortiz-Catalan, R. Brånemark, and B. Håkansson, "BioPatRec: A modular research platform for the control of artificial limbs based on pattern recognition algorithms", Source Code for Biology and Medicine, vol 8, no. 1, pp. 11, 2013.

[9] B. Hudgins, P. Parker, and R. N. Scott, "A new strategy for multifunction myoelectric control," IEEE Trans. Biomed. Eng., vol. 40, no. 1, pp. 82-94, Jan. 1993.

[10] A. Phinyomark, F. Quaine, S. Charbonnier, C. Serviere, F. TarpinBernard, and Y. Laurillau, "EMG feature evaluation for improving myoelectric pattern recognition robustness," Expert Systems with Applications, vol. 40, no. 12, pp. 4832-4840, 2013.

[11] A. Al-Timemy, R. Khushaba, G. Bugmann, and J. Escudero, "Improving the Performance Against Force Variation of EMG Controlled Multifunctional Upper-Limb Prostheses for Transradial Amputees," IEEE Trans Neural Syst Rehabil Eng, vol. 24, 6, 650-661, 2016.

[12] K. Englehart and B. Hudgins, "A robust, real-time control scheme for multifunction myoelectric control," in IEEE Transactions on Biomedical Engineering, vol. 50, no. 7, pp. 848-854, July 2003.

[13] L. J. Hargrove, K. Englehart and B. Hudgins, "A Comparison of Surface and Intramuscular Myoelectric Signal Classification," in IEEE Transactions on Biomedical Engineering, vol. 54, no. 5, pp. 847-853, May 2007.

[14] K. Anam, R. N. Khushaba and A. Al-Jumaily, "Two-channel surface electromyography for individual and combined finger movements," 2013 35th Annual International Conference of the IEEE Engineering in Medicine and Biology Society (EMBC), Osaka, 2013, pp. 49614964.

[15] D. Cai, X. He, and J. Han, "SRDA: An Efficient Algorithm for LargeScale Discriminant Analysis," IEEE Trans. Knowl. Data Eng., vol. 20, pp. 1-12, 2008. 